\section{Messaging Applications: Implications for Healthcare}

In the last two decades, there has been an increase in the ownership of mobile devices like tablets, mobile phones, and computers. The constantly improving networking service worldwide is transforming the healthcare sector with the introduction of eHealth or mHealth - the use of mobile devices for health research and delivery [1]. Some of these include Facebook messenger, iMessage, Koo, Signal, Telegram, WeChat and WhatsApp.

The coronavirus disease 2019 (COVID-19) has disrupted the conventional ways of practicing medicine and demanded socially distant healthcare practice. Recently, the Government of India issued a telemedicine guideline that said, "telemedicine includes all communication channels with the patient that leverage information technology platforms, including voice, audio, text and digital data exchange " [2]. In this situation, WhatsApp came in as a handy tool to consult not only for routine check-ups but also for consultation and monitoring of COVID-19 patients [3]. The World Health Organization (WHO) health alert service also uses WhatsApp to give people up-to-date COVID-19 information.

Various scenarios show that using messaging apps can accelerate communication, streamline workflows, enhance productivity, and improve patient healthcare [4]. They help improve hospital administration efficiency, secure hand-off, faster delivery of laboratory reports, create a discussion thread with seniors and juniors, and collaborate with those outside their workplace easily with seamless integration of health records, and reach healthcare workforce in the peripheral areas [4].

India is a developing nation, and doctors with minimum access to large funds refrain from using professional EMR platforms and use what is freely available and convenient. Platforms like Magpi, Google forms, Survey monkey can be used to collect text and numerical data but when it comes to collecting pictures and videos of the patients, messaging apps are the most convenient option, in addition to being used by researchers to send out survey forms to potential participants.

Despite the benefits, the use of telemedicine has always been concerning due to the lack of clear guidelines in India, unlike some countries like the USA where a healthcare professional can only use a HIPAA compliant platform for telemedicine. Third-party apps like iMessage do not have access to user info, it is not the case with WhatsApp business accounts anymore, making users migrate to other more privacy- oriented appli-cations. Even end to end encryption poses a problem as no data is being stored by WhatsApp (only saved locally on the users' device) which makes it nearly impossible to audit. With messaging applications, it might become very difficult to keep a record of patient data. If either user changes devices, it might lead to complete loss of data unless a full backup is done prior to the shift, which cannot be done remotely. Use of multiple messaging platforms by various professionals may not allow crosstalk between them. Also, app performance is in the control of the company, which may terminate or modify it, based on corporate priorities.

In the current situation, messaging apps can also be used for patient education services like by sharing links to reliable sources of information. The acceptance of messaging is better if the doctor and patients know each other. If it is the first consult, then having a preliminary round of introductions may help build trust. Having ground rules for communication such as when will the clinician respond and the charges for communication via this medium, should be conveyed early on.

Messaging applications can be used in the future for regular follow up of patients. With the development of patient support groups for chronic as well as rare disease using messaging groups would be of excellent use. As capabilities of messaging apps get enhanced, including artificial intelligence, there may be possibilities and threats, which are difficult to foresee presently.

Acknowledgment: Dr Binoy Shah contributed to the writing of the manuscript.

Somashekhar Nimbalkar, * Priyanka Parikh Department of Neonatology, Pramukhswami Medical College, Bhaikaka University, Karamsad 388325 ,

Gujarat.

*somu_somu@yahoo.com

\section{REFERENCES}

1. Langford AT, Solid CA, Scott E, et al. Mobile phone ownership, health apps, and tablet use in US adults with a self-reported history of hypertension: Cross-sectional study. JMIR Mhealth Uhealth [Internet].2019;7:e12228.

2. National Medical Commission. Telemedicine Practice Guidelines. NMC, 2020. Accessed June 2, 2021. Available from: https:/ /www.mohfw.gov.in/pdf/Telemedicine.pdf

3. Sabýrlý R, Karsli E, Canacik O, et al. Use of WhatsApp for polyclinic consultation of suspected patients with COVID-19: Retrospective case control study. JMIR Mhealth Uhealth [Internet]. 2020;8:e22874.

4. Opperman CJ, Vuuren MJ van. WhatsApp in a clinical setting: The good, the bad and the law. South African Journal of Bioethics and Law. 2015;11:102-103.

5. Parikh PA, Shah BV, Phatak AG, et al. COVID-19 pandemic: knowledge and perceptions of the public and healthcare professionals. Cureus [Internet].2020;12:e8144. 